\title{
Mergers of Luminous Early-Type Galaxies
}

\author{
Z. L. Wen ${ }^{1}$, J. L. $\operatorname{Han}^{1}$, and F. S. Liu ${ }^{2}$ \\ ${ }^{1}$ National Astronomical Observatories, Chinese Academy of Sciences, China \\ ${ }^{2}$ College of Physics Science and Technology, Shenyang Normal University, China
}

Galaxy mergers play an important role in many astrophysical processes, such as growth of massive galaxies, triggering AGN, formation of supermassive black hole (SMBH) binaries, and gravitational wave $(\mathrm{GW})$ radiation. Merger rate is one of key quantities for these studies. Previous studies show that the pair fraction varies in a range of $1 \%-10 \%$ in the redshift range of $z=0.2-1.2$. These merger rates are usually calculated from projected close pairs, and very few previous authors have carefully checked the merging fraction of a large sample of pairs. Hence, either small samples or contamination by unphysical pairs introduces large uncertainties. The merger rate should be determined using the fraction of physically merging galaxies, rather than the fraction of galaxies in projected close pairs.

To get an accurate merger rate in the local universe, we constructed a complete pair sample of luminous early-type galaxies with $z<0.12$ using SDSS data with $13.5<r<$ $17.5 \mathrm{mag},(u-r)>2.2$ and $(g-r)>0.7, M_{r}<-21.5 \mathrm{mag}$, and projected separations in the range $7<r_{p}<50 \mathrm{kpc}$. From 87,889 luminous early-type galaxies, 1209 pairs were selected. We then extracted the SDSS $r$-band images for all selected pairs, subtracted the sky background, and applied the GALFIT package (Peng et al. 2002) to account for the smooth symmetric components of the luminosity distribution in the images. Quantitative identification for interaction signatures from the residual images suggests that 249 pairs $(21 \%)$ are merging. Considering the total number of luminous early-type galaxies, we find that about $0.8 \%$ of the galaxies are merging. We adopt an average merging timescale of $0.3_{-0.1}^{+0.2}$ Gyr (Bell et al. 2006) and find the comoving volume merger rate $R_{\mathrm{g}}=(1.0 \pm$ $0.4) \times 10^{-5} \mathrm{Mpc}^{-3} \mathrm{Gyr}^{-1}$.

$\mathrm{SMBH}$ binaries can be formed in galaxy mergers. From identified mergers, we obtain the chirp mass distribution of SMBH binaries following $\Phi\left(\log M / M_{\odot}\right)=(21.7 \pm 4.2)-$ $(3.0 \pm 0.5) \log M / M_{\odot}$. A large number of SMBH coalescences generate a stochastic $\mathrm{GW}$ background at frequency $10^{-9}-10^{-7} \mathrm{~Hz}$, which is a promising GW source for the pulsar timing array. Following Jaffe \& Backer (2003), we find that the strain spectrum of the GW background is $h_{c}(f) \sim 10^{-15}\left(f / \mathrm{yr}^{-1}\right)^{-2 / 3}$, an order of magnitude greater than previous estimates. Details are provided by Wen et al. (2009).

\section{References}

Bell, E. F., et al. 2006, ApJ, 640, 241

Jaffe, A. H. \& Backer, D. C. 2003, ApJ, 583, 616

Peng, C. Y., Ho, L. C., Impey, C. D., \& Rix, H.-W. 2002, AJ, 124, 266

Wen, Z. L., Liu, F. S., \& Han, J. L. 2009, ApJ, 692, 511 